\title{
LA TRADUCCIÓN ENTRE LA MiSERIA Y EL ESPLENDOR: ESTUDIO DE LA RECEPCIÓN DE LA CONCEPCIÓN ORTEGUIANA DE LA TRADUCCIÓN EN EL CONTEXTO TRADUCTOLÓGICO DEL ESPAÑOL
}

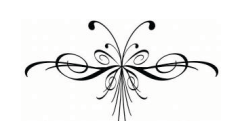

MARÍA DEL PILAR ORdóÑEz LÓPEZ

Resumen: En 1937 Ortega y Gasset publicó su ensayo Miseria y esplendor de la traducción, en el que, desde una perspectiva filosófica, reflexiona sobre la traducción. Desde entonces este ensayo se ha convertido en una referencia prácticamente obligada en la literatura traductológica, especialmente en el contexto traductológico del español. Prueba de la repercusión que este ensayo ha tenido son los distintos estudios en los que se aborda su análisis. En este trabajo nos proponemos analizar dichos trabajos con el objetivo de, por un lado, evaluar en profundidad la recepción que ha tenido el ensayo y la vigencia de la doctrina orteguiana sobre la traducción; $\mathrm{y}$, por otro, valorar hasta qué punto se ha indagado en los planteamientos orteguianos desde una perspectiva integradora, lo cual se resulta indispensable, dada la intertextualidad que caracteriza la obra del filósofo.

Palabras clave: Miseria y esplendor de la traducción; estudios de traducción; Ortega y Gasset.

\begin{abstract}
In 1937 Ortega y Gasset published his essay The Misery and Splendour of Translation, in which he reflects on translation from a philosophical perspective. Since then, that essay has practically become an obligatory reference in the Translation Studies literature, especially within the Spanish-speaking world. The fact that there are several studies aimed at the analysis of that essay confirms the impact that Ortega's work has had. In this article such studies are analysed in order to, on the one hand, provide an in-depth evaluation of the essay's reception and the validity of Ortega's thoughts about translation; and, on the other, to assess to what extent Ortega's ideas about translation have been examined from a comprehensive perspective, which, given the intertextuality of his work, is indispensable in order to provide a complete picture of his vision of translation.
\end{abstract}

Keywords: The Misery and Splendour of Translation; Translation Studies; Ortega y Gasset. 
$\mathrm{L}$ as múltiples referencias al ensayo Miseria y esplendor de la traducción (1937) en la traductología nos llevaron hace unos años a profundizar en el estudio de la influencia de Ortega y Gasset en la reflexión traductológica (Ordóñez López, 2006 y 2009b). En estos trabajos, estudiamos la divulgación del ensayo a través de sus traducciones a diferentes lenguas y su recopilación en las distintas antologías de la traducción, y rastreamos la presencia de la concepción orteguiana de la traducción en la literatura traductológica desde la aparición de la Traductología como disciplina autónoma hasta los primeros años del siglo XXI.

La labor de análisis llevada a cabo permite abundar en la concepción orteguiana de la traducción desde la contextualización del ensayo en la totalidad de la reflexión del filósofo en torno al lenguaje, imprescindible dada la intertextualidad y el constante diálogo interno que caracteriza la obra de Ortega (Ordóñez López, 2009b, p. 41-122). En segundo lugar, el estudio de la divulgación del ensayo, tanto mediante su traducción como a través de su recopilación en las antologías de la traducción, pone de manifiesto su dispar difusión —la primera traducción al alemán, de las varias versiones y ediciones existentes, surge en ya en 1950, mientras que habríamos de esperar al 2004 para la primera traducción al francés- entre el ámbito germánico y el francófono (Ordóñez López, 2006, p. 165-168); por otra parte, la inclusión, al menos parcialmente, del ensayo orteguiano en mayoría de las antologías de la traducción de ámbito general, indica, de acuerdo con los criterios de selección expresados por los propios antólogos, que se trata de una reflexión teórica representativa en la historia de la traducción y que constituye una aportación valiosa como guía de una doctrina, el enfoque hermenéutico de la traducción (Ordóñez López, 2009b, p. 134-146). Finalmente, el análisis de la vigencia de las ideas orteguianas sobre la traducción en la traductología revela que el ensayo Miseria y esplendor de la traducción es una presencia constante en la literatura traductológica occidental durante el periodo analizado, si bien la influencia de la visión de la traducción es heterogénea: mientras que el ensayo orteguiano es, en numerosas ocasiones, objeto de estudio por parte de diversos teóricos (Reiss, 1986; Macola, 1997; Ortega Arjonilla, 1998; Santoyo, 1999; Montezanti, 2000; Arbea, 2001; López Folgado, 2002; Carpintero, 2006; y Bosak, 2008) y en torno a él se articula la reflexión de autores contemporáneos (Steiner, 1975; Reiss, 1988, 2000; Nord, 1989, 2003; García Yebra, 1982, 1983, 1994; López García, 1991; Ortega Arjonilla, 1996), otras veces la referencia a Ortega no es sino una breve mención, cuasi obligada, quizá en un intento de conferir rigurosidad al trabajo en cuestión (Ordóñez López, 2009a, p. 206-209 y 251-253). Finalmente, como no podía ser de otro modo, en los trabajos anteriores constatamos que el célebre Miseria y esplendor de la traducción ha sido objeto de algunas críticas (Vázquez Ayora, 1977; Wilss, 1982; Newmark, 1991; Santoyo, 1999; Ruiz Casanova, 2000). Con todo, los resultados obtenidos demuestran que la concepción orteguiana de la traducción ha tenido una repercusión considerable en la reflexión traductológica, y las referencias continuadas al ensayo indican que éste habría alcanzado la condición de clásico de la reflexión traductora (Ordóñez López, 2009b).

En esta contribución nos proponemos profundizar en el análisis de la recepción de la concepción orteguiana de la traducción en el ámbito traductológico del español. Para ello, nos centraremos en los cinco trabajos dedicados al estudio del ensayo que hallamos en este contexto, con el fin de llevar a cabo una 
revisión comparativa de los mismos que nos permita identificar los aspectos que más repercusión han tenido, aquellos que siguen gozando hoy en día de plena vigencia en la reflexión traductológica, y los que han pasado prácticamente desapercibidos. Asimismo, en este trabajo pretendemos evaluar hasta qué punto se ha abordado el estudio de Miseria y esplendor de la traducción desde una perspectiva integradora, que traspasa los límites del ensayo, y que resulta necesaria en el análisis de la obra orteguiana, para evitar interpretaciones sesgadas de su pensamiento.

\section{Desgranando la miseria, aspirando al esplendor}

En el contexto traductológico del español encontramos, como decíamos, cinco trabajos dedicados al análisis de la reflexión orteguiana en torno a la traducción desde diversas perspectivas. En primer lugar, conviene aclarar que entendemos que formarían parte del ámbito traductológico del español, aquellos trabajos cuyos autores trabajan en universidades, institutos, o centros académicos situados en el marco hispanohablante. En orden cronológico, hallamos los siguientes trabajos: Ortega Arjonilla (1998), Santoyo (1999), Montezanti (2000), Arbea (2001) y López Folgado (2002). De estos trabajos, tres se situarían en el contexto peninsular (Ortega Arjonilla, 1998; Santoyo, 1999; y López Folgado, 2002), y los dos restantes (Montezanti, 2000; y Arbea, 2001) son obra de investigadores de Argentina y Chile, respectivamente.

\section{La aportación de Ortega a la traductología en España}

El primero de los trabajos sobre el ensayo Miseria y esplendor de la traducción es "El legado de Ortega y Gasset a la teoría de la traducción en España” (Ortega Arjonilla, 1998), que contiene un estudio de la concepción orteguiana de la traducción y su aportación a la traductología desde un enfoque eminentemente integrador. Ortega Arjonilla destaca la necesidad, derivada de la naturaleza fragmentaria de la producción intelectual del filósofo, de adoptar una perspectiva intertextual al abordar el estudio del ensayo, para así tomar en consideración la totalidad de la reflexión orteguiana sobre el lenguaje, a fin de lograr una visión global y garantizar el completo entendimiento de los planteamientos del filósofo sobre la traducción. De hecho, afirma Ortega Arjonilla, la falta de una visión más amplia, está detrás de las afirmaciones polémicas que se han vertido sobre el ensayo (Ortega Arjonilla, 1998, p. 12). Por ello, este autor se propone abarcar un entorno más amplio que permita completar la visión reducida, resultante de aproximaciones que se limitan a analizar las ideas contenidas en Miseria y esplendor de la traducción sin tomar en consideración ni las circunstancias de Ortega, ni el resto de su pensamiento sobre el lenguaje.

En primer lugar, hemos de ser conscientes de "la vocación de escritor e intelectual comprometido de Ortega”, caracterizada por su afán de "europeizar España” (Ortega Arjonilla, 1998, p. 105). Teniendo en cuenta este propósito, el ensayo Miseria y esplendor de la traducción se ha de entender como un intento de: 
- sistematizar el trabajo de traducción, dándole la seriedad y el rigor que merece;

- sugerir al traductor que salga del ostracismo y de la timidez que lo caracterizan, desde su punto de vista;

- invitar a los escritores y filólogos al cultivo de la traducción, para darle realce y profesionalizar esta práctica de escritura; y

- considerar a la traducción como un género sui generis que merece ser considerado como tal. (ORTEGA ARJONILLA, 1998, p. 103)

Además, Ortega Arjonilla abunda, desde una perspectiva filosófica, en la concepción del lenguaje de Ortega, relacionándola con la traducción e incorporando aspectos fundamentales en la doctrina orteguiana del lenguaje, como la consideración del lenguaje como producto (ergon) y como actividad (energeia), la distinción planteada por el filósofo entre el hablar, el decir y el callar, y la paradoja del decir (Ortega Arjonilla, 1998, p. 104-108):

Se constata así ese difícil equilibrio que toda lengua mantiene entre lo que se dice y lo que se calla, entre lo explícito y lo sobreentendido o inentendido, y se justifica, de esta forma, que la traducción nunca puede ser mera transposición de estructuras gramaticales, sino mucho más que esto. De tal forma que hemos de aceptar que en la traducción, no sólo confluyen dos sistemas lingüísticos, sino dos sistemas socioculturales y dos formas de vida. (ORTEGA ARJONILLA, 1998, p. 107).

Ortega Arjonilla identifica tres afirmaciones fundamentales en la concepción orteguiana de la traducción, a saber, a) la existencia de distintas versiones posibles de un mismo texto; b) la polarización hacia la versión extranjerizante, "discutible" si nos situamos en otros campos de la práctica profesional de la traducción diferentes de la traducción literaria o filosófica; y c) la propuesta orteguiana de la traducción "fea”. Asimismo, tal y como se indica en el título del trabajo, este autor establece una clasificación de las aportaciones de Ortega a la traductología, en la que distingue tres perspectivas distintas: desde una perspectiva teórica, "la consideración de la traducción dentro del marco más amplio de la comunicación humana” (Ortega Arjonilla, 1998, p. 115), en la que intervienen los factores hablar-decir-callar; desde una perspectiva intelectual, Ortega "propone una elevación de la autoestima del traductor" (Ortega Arjonilla, 1998, p. 115); y desde una perspectiva práctica, si bien los planteamientos orteguianos "deben circunscribirse fundamentalmente al ámbito de los textos literarios y filosóficos, no dejan de ser relevantes para el traductor en la actualidad” (Ortega Arjonilla, 1998, p. 115). Además, añade Ortega Arjonilla, el filósofo “aborda ya la traducción desde una perspectiva 'traductológica', aunque esta denominación sea posterior a la época en la que se escribió este ensayo sobre traducción” (Ortega Arjonilla, 1998, p. 105). Ahora bien, señaladas las aportaciones fundamentales de la reflexión orteguiana en torno a la traducción, advierte:

No podemos pretender, a estas alturas, que Miseria y esplendor de la traducción se constituya en un manual de Traductología. Sin embargo, su valor está en las interpretaciones que hace y en las soluciones que apunta en torno al mundo de la traducción, teniendo en cuenta que su perspectiva es especulativa, filosófica. (Ortega Arjonilla, 1998, p. 114) 


\section{El desmerecido impacto de Miseria y esplendor de la traducción}

Santoyo (1999) adopta un punto de vista crítico hacia el ensayo orteguiano y en su trabajo "En torno a Ortega y Gasset: Miseria y esplendor de la reflexión traductora" ${ }^{1}$ presenta sus discrepancias en torno a la reflexión orteguiana sobre la traducción, con el fin de cuestionar el impacto que ha tenido $\mathrm{Mi}$ seria y esplendor de la traducción en los estudios de traducción.

Santoyo comienza por revisar las circunstancias personales e intelectuales de Ortega en la época previa a la publicación del ensayo, dedicando especial atención a la actitud del filósofo hacia la traducción, a través de las referencias a ésta contenidas en diversas epístolas remitidas por el propio filósofo. De acuerdo con este autor, Ortega no se había interesado particularmente por la traducción hasta la publicación del ensayo (Santoyo, 1999, p. 238), más allá de la consideración de esta actividad como un modo de obtener algunos ingresos suplementarios durante su estancia en Alemania, pese a no manejar aún la lengua alemana con fluidez (Santoyo, 1999, p. 239). Del mismo modo, señala Santoyo, el ensayo Miseria y esplendor de la traducción fue escrito como pago por la ayuda económica recibida en Argentina durante su exilio.

Desde el momento de su publicación, según Santoyo, el ensayo se ha convertido en referencia obligada para "cuantos en España e Hispanoamérica han escrito sobre este tema” (1999, p. 241), logrando, además, reconocimiento en el contexto internacional:

Lo cierto es que, con críticas o sin ellas, el eco nacional e internacional de Miseria y esplendor de la traducción ha sido considerable. Se ha traducido al menos cuatro veces al alemán [...] En 19992, Rainer Schulte y John Biguenet lo han incorporado en traducción inglesa de Elizabeth G. Miller, y junto a los textos de Nabokov, Benjamin, Jakobson, Valéry, etc. a su libro Theories of Translation [...] No sólo eso, hasta bien avanzados los años ochenta, yo diría que hasta los mismos años noventa, Ortega ha sido de facto el único autor español mencionado, cuando se mencionaba alguno, en las bibliografías internacionales de nuestra disciplina [...]. (Santoyo, 1999, p. 241-242)

No obstante, los teóricos de la traducción más destacados no han tenido en cuenta el ensayo, hecho que cabría atribuir a la "condición menor, muy menor del propio ensayo de Ortega” (Santoyo, 1999, p. 242). Por un lado, afirma Santoyo, la mayoría del ensayo no trata de traducción, sino de "otras cosas"; por otro, al abordar la traducción, los planteamientos de Ortega no son más que una reformulación, en "ropaje, estilo y metáforas orteguianos” de autores alemanes como Schleiermacher, Goethe, Humboldt y Rosenzweig (Santoyo, 1999, p. 243). Además, muestra su desacuerdo en torno a dos cuestiones fundamentales: a) la postura categórica adoptada por Ortega al considerar los dos métodos de traducción propuestos por Scheleiermacher, ya que la propuesta del filósofo resulta "absolutamente reprobable para el 99,9\% de las traducciones que se hacen

\footnotetext{
${ }^{1}$ Este trabajo fue presentado originalmente a modo de ponencia, en la Lección inaugural del curso académico 1994-1995, pronunciada el 26 de octubre de 1994 en la Facultad de Traducción e Interpretación de la Universidad Pompeu Fabra de Barcelona; fue publicado con el mismo título en Barcelona, Universtitat Pompeu Fabra, 1994.
} 
en el mundo, literarias o no” (Santoyo, 1999, p. 246); y b) la consideración del filósofo hacia la figura del traductor²:

Sin duda alguna, es un comentario sorprendente [El traductor suele ser un personaje apocado. Por timidez ha escogido tal ocupación, la mínima]. Si atendemos a su opinión, apocados habrían sido Lutero, san Jerónimo, Unamuno y Quevedo, los cuatro bien notorios por su genio, notorios incluso por su mal genio, pero no desde luego por haber sido en modo alguno pusilánimes; apocados habrían sido también los dos Machado, Salvador de Madariaga, Julio Cortázar, fray Luis de León y Buero Vallejo, Juan Ramón Jiménez, el rey Alfredo de Inglaterra y Alfonso X el Sabio en España, Julio Cortázar, Octavio Paz y Agustín García Calvo..., por sólo citar un pequeño ramillete de personalidades “apocadas”, todas ellas bien conocidas por sus traducciones. (Santoyo, 1999, p. 247)

A pesar de las críticas presentadas, el autor valora positivamente la vinculación que establece el filósofo entre la traducción y el habla, y su llamamiento para renovar el prestigio de la traducción, aspecto en el que, en su opinión, las palabras de Ortega al advertir del nacimiento de una disciplina sui generis han resultado ser proféticas (Santoyo, 1999, p. 249).

\section{El carácter anticipatorio de Miseria y esplendor de la traducción}

En "La justicia de Ortega”, Montezanti (2000) pretende hacer frente al tratamiento injusto que ha sufrido Miseria y esplendor de la traducción en el ámbito traductológico anglosajón, donde el ensayo no ha sido más que "parcamente citado" (Montezanti, 2000, p. 37). Montezanti argumenta a favor del carácter pionero y anticipatorio de la reflexión orteguiana, la cual se habría adelantado a los planteamientos de McFarlane y de autores clásicos como Nabokov, Jakobson y Szondi. Este carácter pionero no se limita al punto de vista cronológico, sino que también se aprecia en un sentido conceptual en lo que respecta a los planteamientos orteguianos sobre la intraducibilidad y su defensa de la traducción extranjerizante (Montezanti, 2000, p. 37).

En primer lugar, el autor establece un paralelismo entre Ortega y Quine en torno a la cuestión de la intraducibilidad:

[...] cuando el ensayista considera la traducción como un afán utópico y la inscribe entre los emprendimientos irrealizables del hombre, prefigura la indeterminación radical de Quine. [...] Al indicar que la lengua no sólo pone dificultades a la expresión, sino que 'estorba la recepción de otros [pensamientos]', la reticencia del bizarro expositor orteguiano adelanta lo que de modo más teórico postula Quine en Palabra y objeto y en La realidad ontológica y otros ensayos. (Montezanti, 2000, p. 3738).

No obstante, existen diferencias entre los dos autores, pues Quine "exalta la noción de la perpetua incertidumbre acerca de la corrección o error de una traducción” (Montezanti, 2000, p. 38), mientras que el filósofo español, en

\footnotetext{
${ }^{2}$ Véase Ordóñez López (2009c) para un estudio más detallado sobre esta cuestión.
} 
su propósito de resaltar la miseria de la traducción para realzar así su posible esplendor, adopta una posición en la cual puede entreverse "no sólo la traducibilidad, sino también entusiasmo y virtud en la tarea” (Montezanti, 2000: 39); Ortega no niega la traducción, "bajo condición de que se tenga de ésta un concepto más afinado que el que se tiene comúnmente”, lo que contrasta con la "posición desesperanzada de Quine” (Montezanti, 2000, p. 39).

La propuesta orteguiana de la traducción extranjerizante se ve también reflejada, según Montezanti, en The Translator's Invisibility de Venuti (1995); ahora bien, pese a que ambos autores se posicionan a favor de la extranjerización, existen diferencias significativas en sus planteamientos. Venuti concibe la extranjerización desde el punto de vista de la sociología de la traducción, mientras que Ortega la sitúa en el orden personal y le concede un valor pedagógico. De esta manera, la propuesta de Venuti supondría una alteración de las relación de poder, de un orden social, y para Ortega implicaría la alteración de un orden personal, del "horizonte cognoscitivo y estético del hombre" (Montezanti, 2000, p. 39-40). Ahora bien, en esencia, ambos autores parten de un sustrato común:

[...] cuando Ortega emprende una cruzada a favor de discernir el sentido histórico y erróneo del hombre, Venuti proclama una resistencia contra el etnocentrismo y el racismo: el lenguaje de Venuti es más incisivo y revela un mayor impacto de las ideologías [...] Venuti es predominantemente rebelde hacia [...] la preponderancia de los Estados Unidos y del Reino Unido [...]. El expositor orteguiano al dirigirse a un “auténtico 'humanismo', haciéndole ver bien de cerca el error que fueron los otros y sobre todo, el error que fueron los mejores” (p. 450), cala más hondo, aunque los resultados de uno y de otro sean análogos. (Montezanti, 2000, p. 40).

La noción orteguiana de traducción fea, que no pretende sustituir ni reemplazar a la obra original, es probablemente "el punto más incisivo del ensayo" (Montezanti, 2000, p. 41), puesto que permite superar las comparaciones en términos de "ganancias y pérdidas" en la traducción, y está también latente en Venuti, si bien éste utiliza un enfoque más técnico y menos vehemente (Montezanti, 2000, p. 41). Asimismo, ambos autores compartirían la intención de otorgar prestigio a la labor del traductor (Montezanti, 2000, p. 41).

En la última parte de su trabajo, Montezanti reflexiona sobre dos de las cuestiones más "paradójicas” del ensayo: la elección de interlocutores franceses para el diálogo, que se sitúan frente a un expositor español; y la participación de distintos interlocutores, siendo éste un procedimiento poco común en este género. En su opinión, la primera cuestión respondería al "prestigio de los lingüistas de origen francés", al "celo proverbial de los franceses por la pureza de su lengua”, así como a la posible intención de Ortega de contrastar sus ideas ante un pueblo a quien "irrita la paradoja” (Montezanti, 2000, p. 42). Con relación al carácter polifónico del ensayo, que crea una suerte de “juego de los espejos”, Ortega podría haberlo utilizado para refugiar en él el carácter explorador del ensayo (Montezanti, 2000, p. 43). 


\section{La visión de mundo y Miseria y esplendor de la traducción}

Arbea (2001) se propone analizar el pensamiento de Ortega acerca de la traducción, dedicando especial atención a sus planteamientos sobre cómo ha de ser una buena traducción. El autor comienza por comentar las opiniones más frecuentes en torno a esta cuestión: "por una parte, fiel a su original y, por otra [...] debe estar redactada en buen estilo y ser clara" (Arbea, 2001, p. 195), a lo que, en el caso de la traducción literaria, cabe añadir que "hasta donde sea posible, la belleza del original no debe verse sacrificada” (Arbea, 2001, p. 195-196). Frente a estas opiniones, el ideal orteguiano de la traducción se podría considerar una provocación; sin embargo, en consonancia con el estilo de Ortega, no se trata sino de una paradoja, en la acepción del término empleada por el filósofo - en contraposición a la doxa u opinión comúnmente aceptada.

En su trabajo, este autor ilustra la propuesta de Ortega en cuanto al método o camino de traducir, revisando las distintas posibilidades de traducción de los modos de verbalizar el saludo de los pueblos clásicos según las dos direcciones de traducción presentadas en el ensayo orteguiano (Arbea, 2001, p. 196197):

Las diversas traducciones que he podido consultar de este diálogo de Platón [Ión] coinciden todas en traducir [Ión Iõna khaírein] “iSalud, Ión!”, optando así por la primera alternativa, la que Ortega rechaza por considerarla [...] una imitación del original, una paráfrasis, no una verdadera traducción. Lo que estas versiones traducen [...] es la función del saludo, pero no la forma particular en que Sócrates saluda a Ión [...] En términos más precisos, estas traducciones sacrifican el significado de khaíre, para poder conservar su sentido. (Arbea, 2001, p. 197)

Además, este autor profundiza en las raíces del ideal de traducción de Ortega que, en su opinión, es la consecuencia lógica de la concepción del filósofo de la lengua como visión de mundo, basada en los planteamientos de Humboldt y otros autores del Romanticismo alemán. De este modo, la lengua se concibe como "registro de una determinada perspectiva de la realidad, por un lado, y como órgano generador y modelador del pensamiento, por otro" (Arbea, 2001, p. 201); la propuesta de traducción de Ortega constituye, pues, un medio de acercamiento hacia el otro y de adquirir conciencia histórica de nosotros mismos.

Arbea se hace eco de la falta de seguidores que ha tenido el ideal orteguiano de traducción, algo atribuible, por una parte, a la "larga e ininterrumpida tradición de versiones conscientemente aproximativas [...] que, más que reproducir en todos sus detalles un original, se habían propuesto ser su artística recreación” (Arbea, 2001, p. 201); y, por otra, a "una superficial comprensión del pensamiento del pensador español” (Arbea, 2001, p. 201). Si toda traducción es necesariamente parcial, la propuesta de Ortega permite conservar su alteridad principal, lo más importante para el filósofo, es decir, el contenido, y, según el autor, estas traducciones podrían muy bien simultanearse con otras de lectura menos exigente (Arbea, 2001, p. 202). Para concluir, el autor insta a los profesionales de la traducción a leer atentamente a Ortega y a reflexionar sobre el mejor modo de traducir, para así poder conseguir el propósito expresado por el filósofo de renovar el prestigio de esta labor. 


\section{Integración de lenguaje y traducción}

En "Unamumo and Ortega's Ideas on Language and Translation" (2002), López Folgado profundiza en el pensamiento en torno al lenguaje y la traducción de estos dos destacados pensadores españoles. Al comienzo de su trabajo advierte de que las reflexiones de estos dos autores son el resultado de la asimilación de los planteamientos de la filología alemana decimonónica, si bien representaron una renovación en el contexto español de la época (López Folgado, 2002, p. 43).

Tras revisar el giro experimentado por la lingüística durante el siglo XX y analizar los conceptos de "lengua” y "raza” en Unamuno, López Folgado se dedica a revisar la concepción de la traducción de Ortega. En primer lugar, este autor destaca el carácter dialéctico y antagónico de la reflexión orteguiana sobre la traducción, que ha sido objeto de interpretaciones erróneas que sólo han tomado en cuenta un extremo de esta oposición, pues, si bien esta actividad es descrita como una "tarea humilde” y "utópica”, al mismo tiempo el filósofo la califica de "exorbitante”. La visión utópica de la traducción está íntimamente relacionada con concepción de la lengua de Ortega, basada en los planteamientos humboldtianos sobre la forma interna de las lenguas. De hecho, la esencia de una lengua se revela a través de la traducción, en la que se pone de manifiesto la falta de equivalencia total (López Folgado, 2002, p.54). Así:

[...] it can be claimed that between two given natural languages there is a never ending bridge where the other end can never be met. [...] this is the moot point of translation, the area of meaning which, in spite of conceptual overlapping in a dictionary, has marginal areas that are too subtle to be shared by speakers of both languages and therefore [...] only somebody who is a perfect bilingual would know about those subtle connotative values of words. (López Folgado, 2002, p. 54)

Tomando en cuenta lo anterior, los planteamientos orteguianos sobre la naturaleza utópica de la traducción constituyen, según este autor, una de las aportaciones más reveladoras sobre la traducción; al mismo tiempo, se expresan, de nuevo, recurriendo a una aparente oposición (López Folgado, 2002, p. 55). El carácter utópico inherente a la traducción no se ha de entender como un argumento para no traducir, dado que "the utopia does not forbid the human endevour aimed at achieving the best of versions when translating” (López Folgado, 2002, p. 55).

Para concluir, aborda la propuesta orteguiana de acuerdo con la cual las traducciones de las obras clásicas se han de llevar a cabo sin consideración alguna por la belleza, sino atendiendo al carácter exótico y distante de las mismas (López Folgado, 2002, p. 55). No obstante, este autor concluye su trabajo poniendo en duda el argumento de que el público de un país agradece este tipo de traducción, la traducción extranjerizante, que resulta, además, ser completamente opuesto a las tendencias actuales: 
Quite on the contrary, the readers would surely be critical, and in fact they are often so, with works that sound unlike native texts. Foreign rings are often unwelcome [;] translators are asked to domesticate foreign accents. A method that today's pedagogic theorists would recommend is quite the opposite to Ortega's idea of good translation. (López Folgado, 2002, p. 55)

\section{La miseria y el esplendor del ensayo orteguiano}

En primer lugar, el simple hecho de hallar cinco estudios dedicados al análisis de la reflexión orteguiana sobre la traducción, es un indicio indiscutible de la influencia que el ensayo Miseria y esplendor de la traducción ha tenido en el ámbito traductológico del español. Al mismo tiempo, vemos que el ensayo ha provocado opiniones encontradas; así, mientras Santoyo (1999) pretende poner de manifiesto la desmesurada repercusión del ensayo, Montezanti (2000) reivindica un mayor reconocimiento hacia los planteamientos del filósofo en torno a la traducción. Ortega Arjonilla (1998) y Arbea (2001) también se hacen eco de esta división de opiniones en torno al ensayo orteguiano, hecho que atribuyen a una interpretación sesgada y aislada de la reflexión sobre la traducción del filósofo. Por ello, ambos autores subrayan la necesidad de contextualizar los planteamientos del ensayo en la totalidad de la doctrina orteguiana sobre el lenguaje, y adoptan en sus respectivos artículos una perspectiva más amplia e integradora, relacionando el pensamiento de Ortega sobre la traducción con las circunstancias personales del filósofo, con su visión del lenguaje y del mundo (Ortega Arjonilla, 1998; Arbea, 2001).

Si atendemos a los propósitos o las motivaciones de los autores de los trabajos analizados, encontramos una variedad de propósitos que van desde la intención por poner de manifiesto la desmesurada influencia del ensayo, debido a su carácter poco innovador, al desconocimiento del filósofo de la realidad de la práctica traductora y a su naturaleza divagadora y especulativa; valorar su aportación a la traductología, abordando el análisis del ensayo desde una perspectiva contextualizada; reivindicar su carácter anticipatorio de la reflexión orteguiana en torno a la traducción, para así hacer frente a la falta de reconocimiento del autor en el ámbito anglosajón; vincular el ideal orteguiano de la traducción su visión del lenguaje y del mundo, a fin de profundizar en sus planteamientos sobre la traducción y justificar su postura; y revisar su visión del lenguaje, enraizada en los paradigmas del romanticismo alemán, y sus implicaciones para la traducción.

La revisión de los trabajos aquí reseñados desde un punto de vista contrastivo permite identificar los aspectos de la reflexión orteguiana que más han acaparado la atención de los teóricos de la traducción. En los cinco trabajos, desde perspectivas distintas, se recoge la propuesta de Ortega de encarecer la labor de traducir, de renovar el prestigio de la traducción. Esta propuesta constituye una de las reivindicaciones más explícitas y rotundas de un mayor reconocimiento hacia la figura del traductor, sobre todo en el momento de la publicación del ensayo, por lo que en este punto resulta pionero ante aproximaciones más recientes que reclaman una mayor visibilidad del traductor (Venuti, 1995; Arrojo, 1995; Leech, 2005; entre otros) que gozan de plena vigencia en la reflexión traductológica contemporánea. Otro de los aspectos de la visión orteguiana 
de la traducción reflejado en todos los trabajos analizados es la consideración de la traducción dentro del marco de la comunicación humana: el paralelismo entre traducir y hablar establecido en Miseria y esplendor de la traducción, permite abordar la traducción desde una perspectiva más amplia, accediendo así a toda su complejidad. El establecimiento de este paralelismo o, lo que es más, la equiparación del traducir con el hablar es una las aportaciones más significativas de la reflexión orteguiana sobre la traducción. Las miserias de la traducción, no son, por tanto, exclusivas de esta actividad, ni atribuibles únicamente al personaje del traductor; son inherentes a la comunicación humana, a la naturaleza del lenguaje, y a la propia naturaleza del hombre. Finalmente, la defensa categórica de Ortega de la traducción "fea”, su férrea defensa de la posteriormente denominada "extrajerización”, es otra cuestión presente en los cinco trabajos analizados. La postura de Ortega en lo que respecta al método de traducir es completamente opuesta a los posicionamientos imperantes en los enfoques traductológicos contemporáneos y resulta, como se apunta en los trabajos analizados, poco aplicable a la realidad profesional de la traducción, algo de lo que, como señala Montezanti (2000, p. 41), probablemente era bien consciente el propio Ortega. No obstante, como se afirma en algunos de estos trabajos (Arbea, 2001; Montezanti, 2000), la propuesta de Ortega en este punto no es sino consecuencia lógica de su visión del lenguaje y de su concepción del esplendor de la traducción, que pasaría por favorecer la transmigración del hombre hacia la postura del otro. En este sentido, el esplendor de la traducción pasa también por ser el instrumento a través del cual es posible liberar al hombre de la esclavitud que le impone su lengua, siendo necesario para ello la imposición de otros cuadros y rutas mentales a través de una versión extranjerizante, que, siguiendo este planteamiento, no habría de reemplazar en modo alguno a la obra original.

Otros aspectos que han acaparado la atención de los teóricos son los planteamientos orteguianos sobre los utopismos (Montezanti, 2000; López Folgado, 2002); su afirmación de la existencia de diferentes versiones de un mismo original (Ortega Arjonilla, 1998; Montezanti, 2000; Arbea, 2001); o el papel clave desempeñado por el silencio en la doctrina orteguiana sobre la traducción (Ortega Arjonilla, 1998), si bien en los trabajos analizados este elemento no recibe la atención que merece, si tenemos en cuenta que constituye uno de los elementos fundamentales en la teoría del decir de Ortega ${ }^{3}$, que a su vez se sitúa a la base de su concepción sobre la traducción.

Resulta igualmente llamativo ver cómo parecen haber pasado desapercibidas algunas cuestiones que se plantean en el ensayo, tales como la distinción, ya esbozada y formulada en términos más bien intuitivos, de diferentes modalidades de traducción, articulada a través de la oposición entre lengua y pseudolengua, o terminología (Ortega y Gasset, 1983, V, p. 485); la caracterización orteguiana de la figura del traductor ${ }^{4}$, que habría de entenderse como una provocación, como un intento, en palabras de Arbea (2001, p. 196), de confrontar al público en general con la paradoja (entendida como oposición a la doxa, u opinión comúnmente aceptada), sólo mencionada en la crítica de Santoyo (1999, p. 247) y de forma más indirecta en López Folgado (2002, p. 54); la con-

\footnotetext{
${ }^{3}$ Véase Marco Furrasola (2002) para un análisis extenso y profundo de la concepción orteguiana del silencio.

${ }^{4}$ Véase Ordóñez López (2009c).
} 
sideración de la traducción como un instrumento de actualización, y por tanto, de revitalización de las obras clásicas, a través de la realización de distintas retraducciones de un original. En la misma línea, la estructuración del ensayo, ciertamente poco común para este formato, sólo es abordada por Montezanti (2000); sin embargo la utilización de ese escenario, el Colegio de Francia, y del modo expositivo del diálogo, son también factores relevantes a la hora de analizar el ensayo. Por otra parte, como comenta Montezanti (2000, p. 38) y planteábamos en un trabajo anterior (Ordóñez López, 2009a, p. 82-84), Ortega se opone en su ensayo a los planteamientos de algunos de los lingüistas más influyentes de la época (Meillet, Vendryes), aunque no son estos los únicos autores que aparecen citados en el ensayo. Miseria y esplendor de la traducción no sólo presenta una gran intertextualidad en lo que respecta a la doctrina orteguiana sobre el lenguaje, como hemos señalado, sino que también contiene un abanico de referencias a figuras intelectuales de la época (por ejemplo, Cantor, Condillac, Rosseau, Jean Baruzi) que, salvo en el caso de los lingüistas que acabamos de mencionar y de sus antecedentes alemanes (Schleiermacher, Humboldt, Goethe), pasan desapercibidas en los estudios sobre el ensayo reseñados en este trabajo y que podrían aportar más pistas sobre la contextualización y estructuración del ensayo.

Finalmente, con relación a la intertextualidad y el carácter fragmentario de la obra orteguiana, pese a que en algunos de los trabajos analizados los autores se proponen explícitamente abordar el pensamiento orteguiano sobre la traducción de forma contextualizada para así poder acceder a una visión integradora y abarcar su concepción de la traducción en toda su complejidad, falta aún profundizar en algunas cuestiones que se plantean en el ensayo de manera más o menos explícita y que aparecen también en otros trabajos. Entre ellas cabría mencionar la reflexión orteguiana sobre el estilo y los géneros literarios, cuestiones presentes en el ensayo Miseria y esplendor de la traducción que el filósofo desarrolla de manera fragmentada en otros trabajos, como El hombre y la gente y Meditaciones del Quijote; las diferencias entre lenguas y su jerarquía son objeto también de referencias más o menos desarrolladas en el Comentario al Banquete de Platón, Gracia y desgracia de la lengua francesa, Epílogo para ingleses o Meditación del pueblo joven; la dimensión circunstancial del lenguaje, que el filósofo aborda en diversos ensayos como El hombre y la gente o Meditación del pueblo joven, y que vendría a completar su teoría del decir; o su pensamiento sobre los utopismos, que se retoma en obras como el Comentario al Banquete de Platón o Axiomática para una nueva Filología.

\section{Reflexiones finales}

Los trabajos analizados permiten alcanzar un conocimiento más profundo de la visión orteguiana de la traducción así como del impacto que ésta ha tenido en la reflexión traductológica. Ahora bien, con frecuencia, pasa desapercibida en mayor o menor medida, quizá por considerarse sobreentendida, la actitud general del filósofo en su exposición y el carácter general del ensayo, en el que, utilizando la dialéctica como instrumento, Ortega pretende provocar, confrontar la opinión común. Para ello, recupera algunas de las concepciones erróneas y los mitos más extendidos y arraigados sobre la traducción: el celebérrimo traduttore, traditore, la idea de la traducción como la mágica transustanciación 
de la obra original en otra obra, la falta de reconocimiento y hasta de conciencia sobre la existencia de la figura del traductor.

Ortega concibe la traducción como un instrumento pedagógico de enorme valor, pues a través de ella el hombre puede adquirir conciencia histórica de sí mismo, ampliar su visión del mundo, aprender de los errores cometidos por las civilizaciones que nos precedieron. La traducción es un medio de enriquecimiento, una manera de acceder a los "secretos" de los otros. Es, sin duda, una aproximación humanista, que choca con los enfoques, cada vez más comunes en la literatura traductológica, que enfatizan aspectos más técnicos, más especializados, más aplicados.

Por otro lado, son pocos los trabajos que han acaparado tanta atención en el contexto traductológico del español. Aún así, como diría el filósofo con relación a la traducción, es imposible acceder desde una única perspectiva a todas las dimensiones, connotaciones e implicaciones de los planteamientos de Ortega en Miseria y esplendor de la traducción; rescatando las palabras de Marías, el pensamiento de Ortega es como "los icebergs, de los cuales emerge la décima parte de manera que sólo se puede ver su realidad íntegra buceando” (Marías, 1986, p. 13). Quizá sólo a través del estudio poliédrico y contextualizado del ensayo podamos alcanzar a comprender su visión de la traducción en toda su complejidad, estando así en posición de descubrir el esplendor de la reflexión orteguiana sobre la traducción.

María del Pilar Ordóñez López mordonez@uji.es Profa. Dra., Universitat Jaume I, Castellón de la Plana 


\section{Referencias bibliográficas}

ArbeA, Antonio. Traducción y visión de mundo. Onomázein, Revista de Lingüística, Filología y Traducción, Santiago de Chile, n. 6, p. 195-202, 2001.

Arrojo, Rosemary. The "Death" of the Author and the Limits of the Translator’s Visibility. En: Mary Snell-Hornby, Zuzana Jettmarová y Klaus Kaindl (eds.). Translation as Intercultural Communication. Amsterdam/Philadelphia: John Benjamins, 1995, p. 21-32.

BosAK De Figueiredo, Joana. Weltliteratur y traducción: Utopía y posibilidad. Las ideas de Goethe y de Ortega y Gasset, y la práctica de Larbaud. Transfer, Revista electrónica sobre traducción e interculturalidad, Barcelona, Vol. III (1), p. 38-46, 2008.

Carpintero Ortega, Rafael. Ortega y la traducción. Vasos Comunicantes, Revista de ACE Traductores, Madrid, n. 34, p. 19-24, 2006.

GARCíA YeBrA, Valentín. Teoría y práctica de la traducción. 2 vols. Madrid: Gredos, 1982. 874p.

. En torno a la traducción. Teoría, crítica, historia. Madrid: Gredos, 1983. 382p.

. Traducción: historia y teoría. Madrid: Gredos, 1994. 464p.

LEECH, Wendy. The Translator's Visibility: An Investigation into Public Perceptions of the Translator and How to Raise the Translator's Status in Society. 2005. 100p. Master's Dissertation, Imperial College, The University of London, London.

LÓPEZ FolgADO, Vicente. Unamuno and Ortega's Ideas on Language and Translation. Hikma, Estudios de Traducción, Córdoba, n. 1, p. 43-55, 2002.

LÓPEz GARCíA, Dámaso. Sobre la imposibilidad de la traducción, Albacete: Universidad de Castilla-La Mancha, 1991. 227p.

Macola, Erminia. Stile di pensiero e stile letterario: Ortega in traduzione. En: Erminia Macola y Adone Braudalise (eds.). Ortega y Gasset, J. Vives o L’intelletuale, Padova: Esedra, 1997. p. 123-149.

Marco Furrasola, Ángeles. Una hermenéutica del silencio en Ortega. Revista de Estudios Orteguianos, Madrid, n. 4, p. 89-110, 2002.

MARÍAs, Julian. Introducción. En: Ortega y Gasset, José. La rebelión de las masas (con prólogo para franceses, un epílogo para ingleses y un apéndice: Dinámica del tiempo). Madrid: Espasa-Calpe, 1986. p. 9-31.

Montezanti, Miguel. (2000), La justicia de Ortega. Vasos Comunicantes, Revista de ACE Traductores, Madrid, n. 16, p. 37-44, 2000.

Newmark, Peter. About Translation. Clevedon: Multilingual Matters, 1991. 208p.

NoRD, Christiane. Loyalität statt Treue. Vorschläge zu einer funktionalen Übersetzungstypologie. Lebende Sprache, Zeitschrift für fremde Sprachen in Wissenschaft und Praxis, Leipzig, n. 3, p. 100-105, 1989.

. Function and Loyalty in Bible Translation. En: María Calzada Pérez (ed.). Apropos of Ideology: Translation Studies on Ideology-Ideologies in Translation Studies, Manchester: St. Jerome, 2003. p. 89-113. 
ORDÓÑEZ LÓPEZ, Pilar. Miseria y esplendor de la traducción. La influencia de Ortega y Gasset en la traductología contemporánea occidental. 2006. 349p. Tesis doctoral. Universidad de Granada, Granada.

. Miseria y esplendor de la traducción. La influencia de Ortega en la traductología. Castellón de la Plana: Universitat Jaume I, 2009a. 284p.

. The Misery and Splendour of Translation: a Classic in Translation Studies. SKASE, Journal of Translation and Interpretation, Prešov, Vol. 4 (1), p. 53-78, 2009b.

. Aproximación filosófica á figura do traductor: o retratro do tradutor de Ortega y Gasset. Viceversa, Revista galega de traducción, Vigo, n. 15, p. 23-32, 2009c.

Ortega ArJonilla, Emilio. El legado de Ortega y Gasset a la teoría de la traducción en España. En: Miguel Ángel Vega Cernuda (ed.). La traducción en torno al 98. Madrid: Instituto Universitario de Lenguas Modernas y Traductores, 1998. p. 101-116.

. Apuntes para una teoría hermenéutica de la traducción, Málaga: Universidad de Málaga, 1996. 177p.

Ortega Y Gasset, José. Obras completas, 12 vols.. Madrid: Revista de Occidente, 1946-1983.

REISs, Katharina. Ortega y Gasset, die Sprachwissenschaft und das Übersetzen. Babel, Revue internationale de la traduction/International Journal of Translation, FTI, Basilea, n. 4, p. 202-214, 1986.

. (1988), Der Text und der Übersetzer. En: Rainer Arntz (ed.). Textlinguistik und Frachsprache: AILA-Symposion, Hildesheim 13-16 April 1987. Zurich/New York: Gorg Olms Verlag, 1988.

. Translation Criticism: The Potentials and Limitations. Manchester: St. Jerome, 2000. 127p. [traducción de Erroll F. Rhodes].

Ruiz Casanova, José Francisco. Aproximación a una historia de la traducción en España, Madrid: Cátedra, 2000. 535p.

SANTOYO, Julio César. En torno a Ortega y Gasset. Miseria y esplendor de la reflexión traductora. En: Julio César Santoyo. Historia de la traducción: quince apuntes. León: Universidad de León, 1999. p. 237-250.

SteINER, George. After Babel. Aspects of Language and Translation. New York: Oxford University Press, 1975. 507p.

VÁzQuez AyorA, Gerardo. Introducción a la traductología. Washington, D.C.: Georgetown University Press, 1977. 469p.

Venuti, Lawrence. The Translator's Invisibility: A History of Translation. London/New York: Routledge, 1995. 353p.

WiLss, Wolfgang. The Science of Translation: Problems and Methods. Tübingen: Gunter Narr, 1982. 292p. 